ISSN 1855-3966 (printed edn.), ISSN 1855-3974 (electronic edn.)

ARS MATHEMATICA CONTEMPORANEA 22 (2022) \#P1.10

https://doi.org/10.26493/1855-3974.2329.97a

(Also available at http://amc-journal.eu)

\title{
Signed graphs with two eigenvalues and vertex degree five*
}

\author{
Zoran Stanić \\ Faculty of Mathematics, University of Belgrade, \\ Studentski trg 16, 11000 Belgrade, Serbia
}

Received 6 May 2020, accepted 13 July 2021, published online 17 May 2022

\begin{abstract}
It is known that a signed graph with exactly 2 eigenvalues must be regular, and all those whose vertex degree does not exceed 4 are known. In this paper we characterize all signed graphs with 2 eigenvalues and vertex degree 5 . We also determine all signed graphs with 2 eigenvalues and 12 or 13 vertices, which is a natural step since those with a fewer number of vertices are known.
\end{abstract}

Keywords: Regular signed graph, adjacency matrix, weighing matrix, bipartite double.

Math. Subj. Class. (2020): 05C22, 05C50

\section{Introduction}

A signed graph $\dot{G}$ is a pair $(G, \sigma)$, where $G=(V, E)$ is a simple graph, called the underlying graph, and $\sigma: E \longrightarrow\{1,-1\}$ is the signature. The number of vertices of $\dot{G}$ is denoted by $n$. The edge set of $\dot{G}$ is composed of subsets of positive and negative edges. Two vertices are positive (resp. negative) neighbours if they are joined by a positive (resp. negative) edge.

The adjacency matrix $A_{\dot{G}}$ of $\dot{G}$ is obtained from the adjacency matrix of its underlying graph by reversing the sign of all $1 \mathrm{~s}$ which correspond to negative edges. The eigenvalues of $\dot{G}$ are identified as the eigenvalues of $A_{\dot{G}}$, and they form the spectrum of $\dot{G}$.

We interpret a graph as a signed graph with all the edges being positive and, where no confusion arises, we write ' $\dot{G}$ has $k$ eigenvalues' to mean that $\dot{G}$ has exactly $k$ distinct eigenvalues.

Signed graphs with 2 eigenvalues have been investigated in $[5,7,8,11]$ and some related references. They are known to be regular, moreover every connected signed graph

\footnotetext{
* Research is partially supported by the Serbian Ministry of Education, Science and Technological Development via the Faculty of Mathematics, University of Belgrade.

E-mail address: zstanic@math.rs (Zoran Stanić)
} 
with 2 eigenvalues is strongly regular in the sense of [10]. All signed graphs with 2 eigenvalues and (vertex) degree at most 4 are explicitly determined in [7, 11], and they can also be deduced from the results reported in [8]. In particular, there is an infinite family of those with degree 4. In [8] this family is determined in terms of cyclotomic matrices, and in [11] the same family is determined in terms of signed line graphs. Signed graphs with 2 eigenvalues and at most 11 vertices are reported in [11]. Those whose least eigenvalue is greater than -2 and those which are signed line graphs are also known and can be found in the same reference. There are also some sporadic results related to other classes of signed graphs with 2 eigenvalues [7, 10,11]. Lastly, the Seidel matrix of a simple graph $G$ can be seen as the adjacency matrix of the complete signed graph whose negative edges correspond to the edges of $G$. Accordingly, many results of [2,3] concerning graphs with exactly 2 eigenvalues of the Seidel matrix can be interpreted in the context of signed graphs. Moreover, in this paper we use a similar approach.

Since all signed graphs with 2 eigenvalues and degree at most 4 are known, the next natural step is to consider those with degree 5. In Section 3 we characterize all of them. Moreover, we explicitly determine all except those that belong to some of the two particular infinite families, which remain undetermined but well characterized by certain structural properties.

In Section 4 we determine all signed graphs with 2 eigenvalues and 12 or 13 vertices, which is an extension of the aforementioned result on those with at most 11 vertices.

We start with a preparatory section in which we give some terminology, notation and known results. The paper is concluded by the Appendix that contains certain inequivalent weighing matrices which are frequently used in this paper.

\section{Preliminaries}

We say that a signed graph is connected, regular or bipartite if the same holds for its underlying graph. The negation $-\dot{G}$ is obtained by reversing the sign of every edge of $\dot{G}$. The degree of a vertex is equal to the number of edges incident with it. In particular, the negative degree is the number of negative edges incident with it.

We say that signed graphs $\dot{G}_{1}$ and $\dot{G}_{2}$ are switching isomorphic if there is a monomial $(0,1,-1)$-matrix $P$ such that $A_{\dot{G}_{2}}=P^{-1} A_{\dot{G}_{1}} P$. (We recall that $P^{-1}=P^{\top}$.) In this case we write $\dot{G}_{1} \cong \dot{G}_{2}$. Switching isomorphic signed graphs share the same spectrum and in many considerations they are identified.

The product $\dot{G}_{1} \times \dot{G}_{2}$ of $\dot{G}_{1}$ and $\dot{G}_{2}$ is the signed graph with the vertex set $V\left(\dot{G}_{1}\right) \times V\left(\dot{G}_{2}\right)$ in which two vertices $\left(u_{1}, u_{2}\right)$ and $\left(v_{1}, v_{2}\right)$ are adjacent if and only if $u_{i}$ and $v_{i}$ are adjacent in $G_{i}$, for $1 \leq i \leq 2$. The sign of an edge of the product is the product of the signs of the corresponding edges of $\dot{G}_{1}$ and $\dot{G}_{2}$. The adjacency matrix $A_{\dot{G}_{1} \times \dot{G}_{2}}$ is equal to the Kronecker product $A_{\dot{G}_{1}} \otimes A_{\dot{G}_{2}}$. We recall that the Kronecker product is not symmetric, but the resulting matrices are permutation equivalent, which means that the corresponding signed graphs are switching isomorphic. In particular, $\dot{G} \times K_{2}$ is called the bipartite double (or the bipartite double cover) of $\dot{G}$ and denoted by bd $(\dot{G})$. The bipartite double is always bipartite, and it is connected if and only if $\dot{G}$ is connected and non-bipartite. If $\lambda_{1}, \lambda_{2}, \ldots, \lambda_{n}$ are the eigenvalues of $\dot{G}$ (with possible repetitions), then the eigenvalues of $\operatorname{bd}(\dot{G})$ are $\pm \lambda_{1}, \pm \lambda_{2}, \ldots, \pm \lambda_{n}$.

A weighing matrix $N$ of order $n$ and weight $r$ is an $n \times n(0,1,-1)$-matrix satisfying $N^{\top} N=r I$. We say that two rows of a weighing matrix intersect in $k$ places if their non- 
zero entries match in exactly $k$ positions. Two weighing matrices $M$ and $N$ are said to be equivalent if there are $(0,1,-1)$-monomial matrices $P, Q$ such that $N=P M Q$.

Results reported in the following two sections rely on the classification of weighing matrices of weight 5 and weighing matrices of order 12 obtained by Harada and Munemasa [6]. The first classification is given in the following theorem, while the corresponding matrices can be found in [4, 6]. To make the paper more self-contained we list them in the Appendix. The notation is transferred from the mentioned references.

Theorem 2.1 ([6]). Any weighing matrix of weight 5 is equivalent to a matrix which is a direct sum of an arbitrary number of matrices $W(6,5), W(8,5), W_{12,5}, W_{14,5}, D(16,5)$, $E\left(4 t_{i}+2,5\right), F\left(4 t_{j}+4,5\right)$, where $t_{i}, t_{j} \geq 2$.

Every matrix of the previous theorem determines a bipartite signed graph with the adjacency matrix

$$
\left(\begin{array}{cc}
O & N^{\top} \\
N & O
\end{array}\right),
$$

where $N$ is the matrix in question. Throughout the paper we denote the corresponding signed graphs by $\dot{W}(6,5), \dot{W}(8,5), \dot{W}_{12,5}, \dot{W}_{14,5}, \dot{D}(16,5), \dot{E}(4 t+2,5), \dot{F}(4 t+4,5)$. In other words, a dot indicates that we are dealing with the signed graph. The number of vertices in each of these signed graphs is twice of the first parameter.

\section{Connected signed graphs with 2 eigenvalues and degree 5}

Here is the main result of this section.

Theorem 3.1. A connected signed graph with degree 5 has 2 eigenvalues if and only if it is switching isomorphic to

(i) $K_{6},-K_{6}$,

(ii) one of the bipartite signed graphs $\dot{W}(6,5), \dot{W}(8,5), \dot{W}_{12,5}, \quad \dot{W}_{14,5}, \dot{D}(16,5)$, $\dot{E}(4 t+2,5), \dot{F}(4 t+4,5)$,

(iii) one of the non-bipartite signed graphs illustrated in Figure 1 or a non-bipartite signed graph $\dot{G}$ such that either $\operatorname{bd}(\dot{G}) \cong \dot{E}(4 t+2,5)$ or $\operatorname{bd}(\dot{G}) \cong \dot{F}(4 t+4,5)$,

where, wherever it occurs, the parameter $t$ satisfies $t \geq 2$.

Every signed graph of (ii) or (iii) has the eigenvalues $\pm \sqrt{5}$.

Evidently, a disconnected signed graph with 2 eigenvalues and degree 5 is a disjoint union of connected ones. The proof of the previous theorem is based on the subsequent lemmas. In the first one we consider the case in which the eigenvalues are asymmetric (i.e., they are not equal in absolute value).

Lemma 3.2. Every connected signed graph with degree 5 and 2 asymmetric eigenvalues is switching isomorphic to $K_{6}$ or $-K_{6}$.

Proof. By considering the minimal polynomial we deduce that the eigenvalues must be integral and the negation of their product is equal to the degree, i.e., 5 . This gives just 2 possibilities which further produce the desired solutions. 


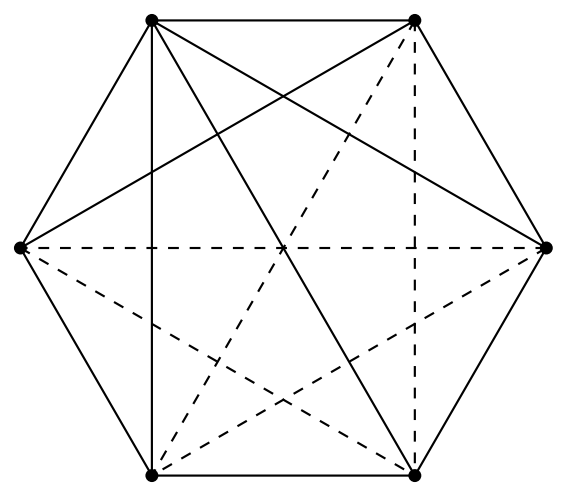

(a)

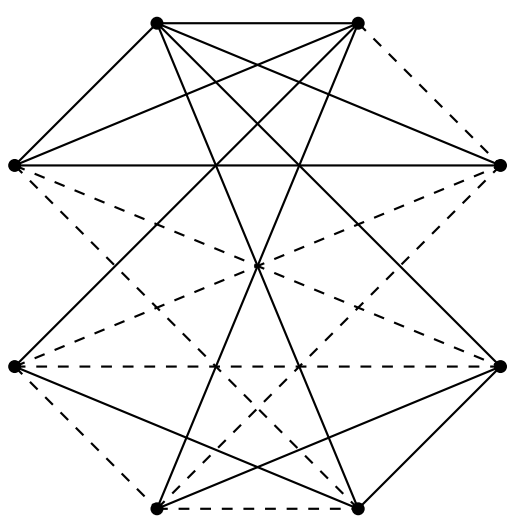

(b)

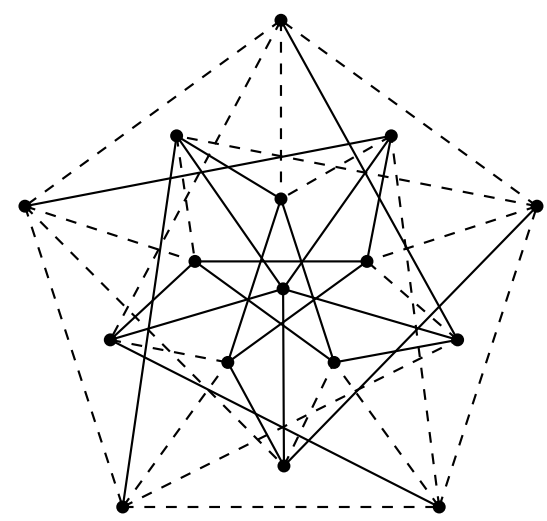

(c)

Figure 1: Signed graphs of Theorem 3.1(iii). In this and the forthcoming figures, negative edges are dashed.

In what follows we assume that the eigenvalues are symmetric. In this case they are $\pm \sqrt{5}$, so our task is to consider all signed graphs with these particular eigenvalues. We first consider the bipartite case.

Lemma 3.3. Every connected bipartite signed graph with eigenvalues $\pm \sqrt{5}$ is switching isomorphic to either $\dot{W}(6,5), \dot{W}(8,5), \dot{W}_{12,5}, \dot{W}_{14,5}, \dot{D}(16,5), \dot{E}(4 t+2,5)$ or $\dot{F}(4 t+4,5)$ (for $t \geq 2$ ).

Proof. Let

$$
A_{\dot{G}}=\left(\begin{array}{cc}
O & M^{\top} \\
M & O
\end{array}\right)
$$

be the adjacency matrix of a signed graph under consideration. It follows that $M$ is a square matrix satisfying $M^{\top} M=5 I$, i.e., $M$ is a weighing matrix of weight 5 . Since $\dot{G}$ is connected, $M$ is equivalent to one of the seven matrices listed in the formulation of this lemma. If we denote this matrix by $N$, we have $M=P N Q$ for some $(0,1,-1)$-monomial 
matrices $P$ and $Q$, which implies

$$
A_{\dot{G}}=\left(\begin{array}{cc}
Q^{\top} & O \\
O & P
\end{array}\right)\left(\begin{array}{cc}
O & N^{\top} \\
N & O
\end{array}\right)\left(\begin{array}{cc}
Q & O \\
O & P^{\top}
\end{array}\right)=\left(\begin{array}{cc}
Q & O \\
O & P^{\top}
\end{array}\right)^{-1}\left(\begin{array}{cc}
O & N^{\top} \\
N & O
\end{array}\right)\left(\begin{array}{cc}
Q & O \\
O & P^{\top}
\end{array}\right),
$$

and the result follows.

The essential part of the proof of Theorem 3.1 refers to the non-bipartite case. Observe that every connected non-bipartite signed graph $\dot{G}$ with eigenvalues $\pm \sqrt{5}$ is extracted from a decomposition of a bipartite one (with the same eigenvalues) having the form $\dot{G} \times K_{2}$, i.e., which is a bipartite double of $\dot{G}$. We remark that not every bipartite signed graph is a bipartite double, and a decomposition does not need to be unique (in the sense that it may produce switching non-isomorphic signed graphs). We also have $\operatorname{bd}(\dot{G}) \cong \operatorname{bd}(-\dot{G})$. In what follows we consider possible decompositions of signed graphs of Lemma 3.3. For this purpose we need the following simple but very useful result. It helps us to determine the common neighbourhood of the particular vertices of $\dot{G}$.

Lemma 3.4. If $W_{1}$ and $W_{2}$ are the colour classes of $\operatorname{bd}(\dot{G})$, then there are bijections $f_{1}: V(\dot{G}) \longrightarrow W_{1}$ and $f_{2}: V(\dot{G}) \longrightarrow W_{2}$ such that $u, v$ are adjacent in $\dot{G}$ if and only if $f_{1}(u), f_{2}(v)$ and $f_{2}(u), f_{1}(v)$ are adjacent in $\operatorname{bd}(\dot{G})$.

Proof. The result follows by definition of the Kronecker product.

In the following two lemmas we consider $\dot{W}(6,5)$ and $\dot{W}(8,5)$. Despite the corresponding results can be obtained by computer search, we give theoretical proofs. They illustrate a technique which is used in the forthcoming considerations.

Lemma 3.5. $\operatorname{bd}(\dot{G}) \cong \dot{W}(6,5)$ holds if and only if $\dot{G}$ is switching isomorphic to the signed graph illustrated in Figure 1(a).

Proof. Since every two rows of $W(6,5)$ intersect in 4 places, we conclude (by Lemma 3.4) that $\dot{G}$ is a complete signed graph with 6 vertices. Since its eigenvalues are $\pm \sqrt{5}$, its adjacency matrix is equivalent to $W(6,5)$, and (up to switching) there is the unique possibility, $W(6,5)$ itself, which leads us to the signed graph of Figure 1(a).

The opposite implication is immediate.

Considering the minimal polynomial, we get that if $\dot{G}$ has the eigenvalues $\pm \sqrt{5}$, then for every pair of its vertices we have

$$
w_{2}(u, v)= \begin{cases}5 & \text { if } u=v \\ 0 & \text { if } u \neq v\end{cases}
$$

where $w_{2}(u, v)$ denotes the difference between the number of 2-walks between $u$ and $v$ which traverse edges of the same sign and the number of 2 -walks between the same vertices which traverse edges of different sign.

We proceed with $\dot{W}(8,5)$.

Lemma 3.6. $\operatorname{bd}(\dot{G}) \cong \dot{W}(8,5)$ holds if and only if $\dot{G}$ is switching isomorphic to the signed graph illustrated in Figure 1(b). 
Proof. Since the first (resp. last) four rows of $W(8,5)$ intersect each other in the first (resp. last) 4 places, from Lemma 3.4, we conclude that the vertices of $\dot{G}$ are partitioned into two sets of equal size in such a way that each vertex of the first set is adjacent to each vertex of the second. We claim that, up to switching, there is a unique distribution of the edges between the vertices of the mentioned sets as given in Figure 1(b), where the vertices of the first set are drawn in left part. Indeed, without loss of generality, we may assume that $\dot{G}$ contains a vertex whose negative degree is 0 (the top-left vertex in the figure), and then every remaining vertex in its set has two positive and two negative neighbours in the other set, which together with (3.1) gives the desired edge distribution. It remains to insert the remaining 4 edges, 2 in each set. Again, on the basis of (3.1) we arrive at the unique possibility, which gives $\dot{G}$.

The uniqueness (up to switching) follows by the way of construction, and the opposite implication is immediate.

Observe that, according to (3.1), every pair of vertices of $\dot{G}$ has an even number of common neighbours. Consequently, every pair of rows of $A_{\mathrm{bd}(\dot{G})}$ intersects in an even number of places. Since the degree is 5 , they intersect in 0,2 or 4 places. In what follows, we consider one particular case. We say that a signed graph is triangle-free if it does not contain a triangle as a subgraph.

Lemma 3.7. If every pair of rows of $A_{\mathrm{bd}(\dot{G})}$ intersects in either 0 or 2 places, then $\dot{G}$ is triangle-free.

Proof. Assume that $\dot{G}$ is not triangle-free. Then, every edge of $\dot{G}$ that belongs to some triangle, in fact, belongs to exactly 2 triangles. Indeed, by (3.1) applied to the vertices incident with such an edge, we conclude that it must belong to an even number of triangles which, by Lemma 3.4 and the assumption of this lemma, makes this number equal to 2 . If so, then $\dot{G}$ contains a tetrahedron, or an octahedron, or an icosahedron as anduced subgraph. Moreover, the octahedron is eliminated immediately since it contains a pair of vertices with 4 common neighbours, which would imply the existence of two rows in $A_{\mathrm{bd}(\dot{G})}$ that intersect in 4 places.

First let $\dot{G}$ contain a tetrahedron, and let its vertices be denoted by $a, b, c, d$. Assume that the negative degree of $a$ is 0 . Under the assumption of this lemma, $a, b$ have no common neighbours outside the fixed tetrahedron, and thus $w_{2}(a, b)=0$ yields $\sigma(b c)=-\sigma(b d)$. Similarly, $w_{2}(a, c)=0$ gives $\sigma(c b)=-\sigma(c d)$, which implies $\sigma(b d)=\sigma(c d)$. But from $w_{2}(a, d)=0$, we get $\sigma(b d)=-\sigma(c d)$, which contradicts the previous equality.

If $\dot{G}$ contains an icosahedron, then it also contains (as an induced subgraph) a pentagon and an additional vertex adjacent to all the vertices of the pentagon. By assuming that the negative degree of the additional vertex is 0 , we arrive at a contradiction in a very similar way as before.

We now eliminate $\dot{W}_{12,5}$ and $\dot{W}_{14,5}$ (as candidates for bd $(\dot{G})$ ) and consider $\dot{D}(16,5)$.

Lemma 3.8. There is no signed graph $\dot{G}$ such that $\operatorname{bd}(\dot{G}) \cong \dot{W}_{12,5}$ or $\operatorname{bd}(\dot{G}) \cong \dot{W}_{14,5}$.

Proof. Assume by way of contradiction that either $\operatorname{bd}(\dot{G}) \cong \dot{W}_{12,5}$ or bd $(\dot{G}) \cong \dot{W}_{14,5}$. In both cases $A_{\mathrm{bd}(\dot{G})}$ satisfies the assumption of Lemma 3.7, and thus $\dot{G}$ is triangle-free, and of course the same holds for its underlying graph $G$. 
The spectrum of the underlying graph of $\dot{W}_{12,5}$ is $\left[ \pm 5,( \pm \sqrt{5})^{6},( \pm 1)^{5}\right]$. Since the nonintegral algebraic conjugates are equal in multiplicity, there is just one candidate for the spectrum of $G$ : $\left[5,( \pm \sqrt{5})^{3},(-1)^{5}\right]$. According to [12], there is exactly one graph with this spectrum (known as the icosahedron), but it contains a triangle - a contradiction.

Similarly, the spectrum of the underlying graph of $\dot{W}_{14,5}$ is $\left[ \pm 5, \pm 3,( \pm(1+\sqrt{2}))^{6}\right.$, $\left.( \pm(1-\sqrt{2}))^{6}\right]$. There is just one candidate for the spectrum of $G$ that passes the numerical condition $\operatorname{tr}\left(A_{G}\right)=0$ : $\left[5,3,1 \pm \sqrt{2},(-1 \pm \sqrt{2})^{5}\right]$. But since in this case we have $\operatorname{tr}\left(A_{G}^{3}\right) \neq 0$, we deduce that $G$ contains a triangle - a contradiction.

Lemma 3.9. $\operatorname{bd}(\dot{G}) \cong \dot{D}(16,5)$ holds if and only if $\dot{G}$ is switching isomorphic to the signed graph illustrated in Figure 1(c).

Proof. Since $A_{\mathrm{bd}(\dot{G})}$ satisfies the assumption of Lemma 3.7, we get that $G$ is triangle-free. The spectrum of the underlying graph of $\dot{D}(16,5)$ is $\left[ \pm 5,( \pm 3)^{5},( \pm 1)^{10}\right]$, which together with $\operatorname{tr}\left(A_{G}\right)=0, \operatorname{tr}\left(A_{G}^{3}\right)=0$, leads to the unique possibility for the spectrum of $G$ : $\left[5,1^{10},(-3)^{5}\right]$. There is exactly one graph with this spectrum known as the Clebsch graph (the underlying graph of the signed graph illustrated in the figure).

In what follows we consider the signature $\sigma$ defined on $E(G)$ which would produce the desired $\dot{G}$. From this point we use a descriptive terminology and refer the reader to follow the corresponding figure. As in the previous proofs, without loss of generality, we assume that (at least) one vertex is not incident with negative edges; let this be the central vertex of the figure, denoted here by $a$. Observe that (in the figure) $a$ is surrounded by the 5 vertices which form a pentagram. Denote the set of these vertices by $P$ and the set of the remaining vertices at distance 2 from $a$ by $Q$. Now, we have the following:

- Every neighbour of $a$ has 2 neighbours in $P$ and exactly one of them is a positive neighbour (otherwise, $w_{2}(a, b) \neq 0$ for at least one $b \in P$ );

- Every neighbour of $a$ has 2 neighbours in $Q$ and exactly one of them is a positive neighbour (for a similar reason);

- For $b, c$ being neighbours of $a$ such that $b, c$ have a common neighbour $d$ in $P$, we have $\sigma(b d)=-\sigma(c d)$. The same holds if $d \in Q$.

Using these conditions we arrive at a unique (up to switching) signature for the edges incident with neighbours of $a$. Since $w_{2}(b, c)=0$ for $b, c \in P$, we get that the edges between the vertices of $P$ have the same sign. If they are positive (as in the figure), then all the remaining (undecided) edges are negative (due to (3.1) applied to non-neighbours of $a$ ), and we get the desired $\dot{G}$. If the mentioned edges are taken to be negative, we arrive at a switching isomorphic signed graph.

The opposite implication is immediate.

We now consider the families of signed graphs illustrated in Figure 2. We first explain their structure. The signed graph of Figure 2(a) has $4 t+2(t \geq 2)$ vertices, and the vertices distinct from $a, b$ are partitioned into the isomorphic blocks with 4 vertices. For example, the vertices $c, d, e, f$ belong to one block. There are no negative edges between two blocks. The signed graph of Figure 2 (b) has $4 t+4(t \geq 2)$ vertices, and is obtained from the previous one by deleting the 4 edges between two blocks, then inserting two vertices adjacent by a positive edge along with the edges between them and each of the 4 vertices of degree 3 of the corresponding blocks in such a way that the negative edges are 


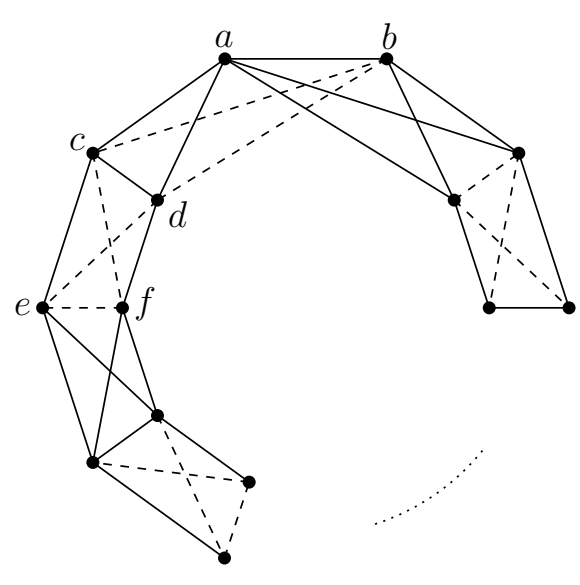

(a)

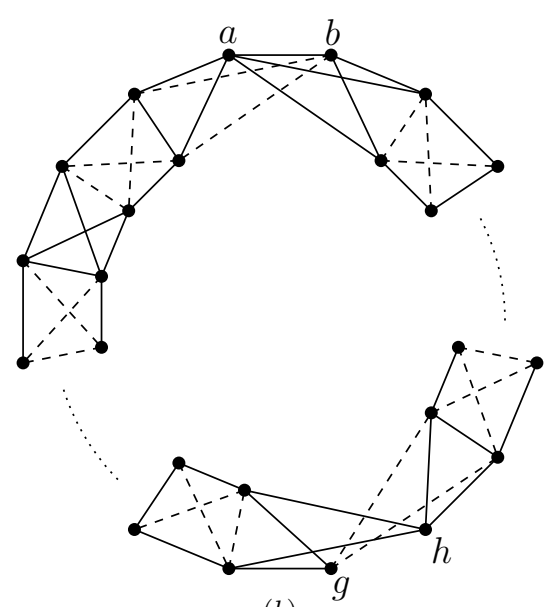

(b)

Figure 2: Signed graphs of Lemma 3.10.

just the two edges between one of the new vertices and the block vertices adjacent by a positive edge. If (in the figure) $a, b$ are transferred from the previous signed graph, then the new vertices are $g, h$.

Lemma 3.10. If $\dot{G}$ is switching isomorphic to the signed graph illustrated in Figure 2(a) (resp. Figure $2(\mathrm{~b}))$, then $\operatorname{bd}(\dot{G}) \cong \dot{E}(4 t+2,5)($ resp. bd $(\dot{G}) \cong \dot{F}(4 t+4,5))$.

Proof. In both cases, $\dot{G}$ is regular of degree 5 and $w_{2}(u, v)=0$ holds for every pair of its distinct vertices, which is verified easily. Therefore, $A_{\dot{G}}^{2}=5 I$, which means that the eigenvalues of $\dot{G}$ are $\pm \sqrt{5}$. Since, obviously, $\dot{G}$ is non-bipartite, its bipartite double is connected and has the same eigenvalues. In other words, $\operatorname{bd}(\dot{G})$ is one of the bipartite signed graphs listed in Lemma 3.3. Now, $\dot{W}(6,5), \dot{W}(8,5)$ are eliminated since bd $(\dot{G})$ has at least 20 vertices, $\dot{W}_{12,5}, \dot{W}_{14,5}$ are eliminated by Lemma 3.8 , and $\dot{D}(16,5)$ is eliminated since, contrary to $\operatorname{bd}(\dot{G})$, it does not contain a pair of vertices with 4 common neighbours. Therefore, either bd $(\dot{G}) \cong \dot{E}(4 t+2,5)$ or bd $(\dot{G}) \cong \dot{F}(4 t+4,5)$. Comparing the numbers of vertices, we deduce that the first possibility occurs precisely if $\dot{G}$ is the first signed graph of the figure.

We prove the main result of this section.

Proof of Theorem 3.1. If the eigenvalues are asymmetric, then Lemma 3.2 leads to (i). If the eigenvalues are symmetric, from Lemma 3.3 we get (ii), while from Lemmas 3.5, 3.6, 3.8, 3.9 we get (iii).

Lemma 3.10 shows that the presence of $\dot{E}(4 t+2,5)$ and $\dot{F}(4 t+4,5)$ in the formulation of Theorem 3.1(iii) is essential. This lemma leads to the question on how we get the signed graphs of Figure 2, and the answer is simple: We arrive at them by following a simple reasoning based on (3.1) and Lemma 3.4. To determine all the signed graphs that can be extracted from decompositions of $\dot{E}(4 t+2,5)$ and $\dot{F}(4 t+4,5)$, one should proceed with the similar reasoning, which in fact becomes complicated especially under the assumption 
that there exist non-adjacent vertices with 4 common neighbours. An example is given in the next section.

\section{Signed graphs with 2 eigenvalues and 12 or 13 vertices}

We have mentioned in Section 1 that signed graphs with 2 eigenvalues and at most 11 vertices are known. Those with at most 10 vertices are obtained by computer search reported in [11]. According to the same reference, there are exactly 2 such signed graphs with 11 vertices up to switching: $K_{11}$ and its negation.

It occurs that, for $n=12$, a computer search which considers all regular signed graphs takes a long time, and so this task requires the application of more sophisticated methods. In what follows we report the results obtained on the basis of a theoretical and computational search on connected signed graphs with 2 eigenvalues and 12 vertices. Let $\dot{G}$ denote such a signed graph and let $\lambda, \mu$ be its eigenvalues. Recall that vertex degree of $\dot{G}$ is $r=-\lambda \mu$.

Assume first that the eigenvalues $\lambda, \mu$ are asymmetric (and then they must be integral). Considering $r$, we easily eliminate the possibility $|\lambda|,|\mu| \geq 3$. If $|\mu|=1$, we arrive at $K_{12}$ and $-K_{12}$. If $\mu=-2$, by taking into account $\operatorname{tr}\left(A_{\dot{G}}\right)=0$ and $\operatorname{tr}\left(A_{\dot{G}}^{2}\right)=r n$, we arrive at the unique possible spectrum $\left[4^{4},(-2)^{8}\right]$. Now, since the least eigenvalue is not less than -2 , the corresponding signed graph is either the signed line graph (in the sense of a definition given in $[1,11]$ ) or the so-called exceptional signed graph. Moreover, it cannot be exceptional since the multiplicity of -2 in every exceptional signed graph is $n-6$, or $n-7$, or $n-8$, as confirmed in [11]. On the other hand, all signed line graphs with 2 eigenvalues are determined in the same reference, and accordingly $\dot{G}$ is switching isomorphic to the line graph of a signed multigraph obtained by inserting a negative edge between every pair of vertices of the complete graph $K_{4}$. For all details (including definition of the signed line graph) we refer the reader to the corresponding reference, but we also illustrate $\dot{G}$ in Figure 3(a). Of course, case $\mu=2$ produces its negation, and we are done.

Assume now that $\lambda, \mu$ are symmetric. Since the case $r \leq 4$ is resolved for every $n$, we may also assume that $r \geq 5$. For $r=5$, we immediately get $\dot{W}(6,5)$ and the signed graph of Figure 2(b) (obtained for $n=12$ ). We redraw this signed graph in Figure 3(b). To conclude the list we consider other $\dot{G}$ 's satisfying $\operatorname{bd}(\dot{G}) \cong \dot{F}(12,5)$. This can be performed either by hand or by a brute force, i.e., a computer search. In either way, we arrive at another solution illustrated in Figure 3(c).

We proceed with $r \geq 6$. According to [6], there are 8 inequivalent weighing matrices of order 12 and weight 6,3 of weight 7,7 of weight 8,4 of weight 9,5 of weight 10 and 1 of weight 11 . They determine all connected bipartite signed graphs with 24 vertices, 2 symmetric eigenvalues and degree $r$, where $6 \leq r \leq 11$. Using the method exploited in the previous section, we consider possible decompositions of each of them. In each case we start from the given weighing matrix (the data on them can be found in [6]), then compute the spectrum of the underlying graph of the corresponding bipartite signed graph, and then compute the spectrum of a putative graph which can be extracted from it.

In this way we arrive at the data of Table 1 . So, the 8 weighing matrices of weight 6 produce the underlying bipartite graphs with 4 distinct spectra (listed in the second column), and each of these spectra gives spectra (listed in the third column) of the underlying graph $G$ of a putative signed graph $\dot{G}$. The remaining weights are considered in the same way, and all the possible spectra of the third column satisfy $\operatorname{tr}\left(A_{G}\right)=0, \operatorname{tr}\left(A_{G}^{2}\right)=12 r$. Note that in one case for weight 8 , there is no spectrum that obeys these numerical conditions. 


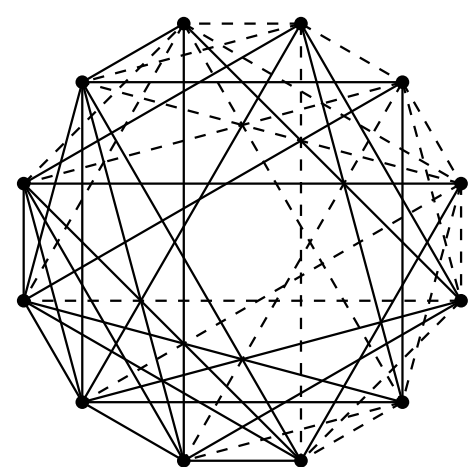

(a)

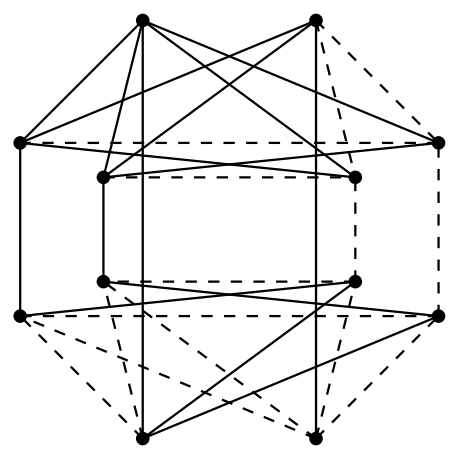

(c)

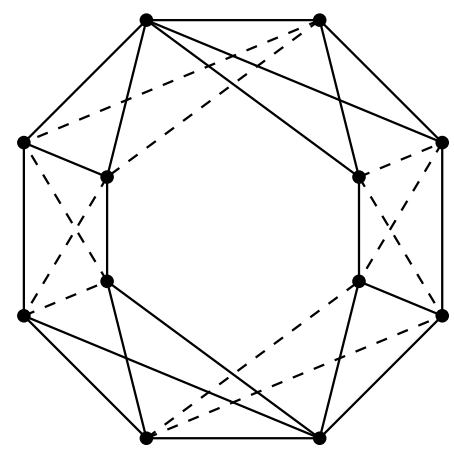

(b)

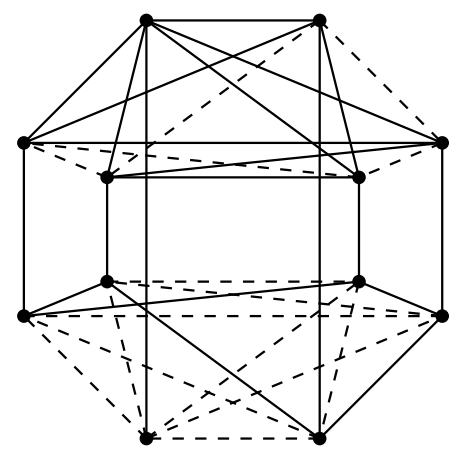

$(d)$

Figure 3: Signed graphs of Theorem 4.1.

The table omits the data for weight 11 , since this case is resolved directly. Namely, if the corresponding bipartite signed graph is a bipartite double of a signed graph $\dot{G}$, then $\dot{G}$ is the complete signed graph with 12 vertices and eigenvalues $\pm \sqrt{11}$, but such a signed graph does not exist, which can be confirmed by examining a list of Seidel's strong graphs - an appropriate reference is [9].

It remains to consider the spectra of the third column. (Observe that they are enumerated by $1-15$ ). First, there is no graph with spectrum $4,5,9,11$ or 14 , which can easily be confirmed by inspecting regular graphs with 12 vertices. There are 2 graphs with spectrum 7 , and each contains pairs of vertices with 5 common neighbours which implies the non-existence of $\dot{G}$ in these cases. There is exactly 1 graph with spectrum $1,10,12,13$ or 15 , and the non-existence of $\dot{G}$ is confirmed either by hand (as these graphs have a high level of symmetry along with a large number of vertices with the same neighbourhood) or by computer search. There are exactly 4 graphs with spectrum 3,1 graph with spectrum 6 and 1 graph with spectrum 8 . The non-existence of $\dot{G}$ is confirmed by computer search. Finally, there is exactly 1 graph with spectrum 2 . By computer search we find exactly 1 (up to switching) $\dot{G}$ - it is illustrated in Figure 3(d); with consistent vertex labellings its 


\begin{tabular}{|c|c|c|}
\hline weight & spectrum of $G \times K_{2}$ & spectrum of $G$ \\
\hline \multirow{4}{*}{6} & $\pm 6,( \pm 3.24)^{3},( \pm 1.24)^{3}, 0^{10}$ & 1. $6,1.24^{3}, 0^{5},(-3.24)^{3}$ \\
\hline & $\pm 6, \pm 4,( \pm 2)^{5}, 0^{10}$ & $\begin{array}{l}\text { 2. } 6,4,0^{5}(-2)^{5} \\
\text { 3. } 6,2^{2}, 0^{5},(-2)^{3},-4\end{array}$ \\
\hline & $\pm 6, \pm 3.86, \pm 2.83,( \pm 2)^{3}, \pm 1.04,0^{10}$ & $\begin{array}{l}\text { 4. } 6,3.86,0^{5},-1.04,(-2)^{3},-2.83 \\
\text { 5. } 6,2.83,1.04,0^{5},(-2)^{3},-3.86\end{array}$ \\
\hline & $\pm 6, \pm 3.46,( \pm 2)^{3}, 0^{12}$ & $6.6,3.46,0^{6},(-2)^{3},-3.46$ \\
\hline \multirow[t]{2}{*}{7} & $\pm 7,( \pm 3)^{3},( \pm 1)^{8}$ & $\begin{array}{l}\text { 7. } 7,1^{5},(-1)^{3},(-3)^{3} \\
8.7,3,1^{2},(-1)^{6},(-3)^{2}\end{array}$ \\
\hline & $\pm 7,( \pm 2.24)^{6},( \pm 1)^{5}$ & 9. $7,2.24^{3},-2.24^{3},(-1)^{5}$ \\
\hline \multirow{4}{*}{8} & $\pm 8,( \pm 4)^{2}, 0^{18}$ & $10.8,0^{9},(-4)^{2}$ \\
\hline & $\pm 8, \pm 3.46,( \pm 2)^{2}, 0^{14}$ & 11. $8,3.46,0^{7},(-2)^{2},-3.46$ \\
\hline & $\pm 8, \pm 4,( \pm 2)^{4}, 0^{12}$ & $12.8,2,0^{6},(-2)^{3},-4$ \\
\hline & $\pm 8, \pm 4,( \pm 2.83)^{2}, 0^{16}$ & \\
\hline 9 & $\pm 9,( \pm 3)^{2},( \pm 1)^{9}$ & $\begin{array}{l}\text { 13. } 9,1^{3},(-1)^{6},(-3)^{2} \\
\text { 14. } 9,3,(-1)^{9},-3\end{array}$ \\
\hline 10 & $\pm 10,( \pm 2)^{5}, 0^{12}$ & $15.10,0^{6},(-2)^{5}$ \\
\hline
\end{tabular}

Table 1: Data for the search on connected non-bipartite signed graphs with 2 symmetric eigenvalues and 12 vertices.

adjacency matrix is

$$
\left(\begin{array}{cc}
W(6,5) & I \\
I & -W(6,5)
\end{array}\right) .
$$

The results are summarized in the following theorem.

Theorem 4.1. A connected signed graph with 12 vertices and degree at least 5 has 2 eigenvalues if and only if it is switching isomorphic to either $K_{12},-K_{12}, \dot{W}(6,5)$, one of signed graphs illustrated in Figure 3 or the negation of the signed graph of Figure 3(a).

Finally, we quickly resolve the case $n=13$.

Theorem 4.2. Every signed graph with 2 eigenvalues and 13 vertices is switching isomorphic to $K_{13}$ or $-K_{13}$.

Proof. Since the number of vertices is odd, the eigenvalues $\lambda, \mu$ must be asymmetric, and then the proof is similar to the proof of Lemma 3.2 or to above consideration related to the asymmetric case for $n=12$. Accordingly, for $|\mu|=1$ we arrive at the desired signed graphs. Up to negation, the remaining possibilities are $\mu=-2, \lambda \in$ $\{3,4,5,6\}$ and $(\mu, \lambda)=(-3,4)$, and both are eliminated by the conditions $\operatorname{tr}\left(A_{\dot{G}}\right)=0$, $\operatorname{tr}\left(A_{\dot{G}}^{3}\right)=13 r$.

\section{ORCID iDs}




\section{References}

[1] F. Belardo and S. K. Simić, On the Laplacian coefficients of signed graphs, Linear Algebra Appl. 475 (2015), 94-113, doi:10.1016/j.laa.2015.02.007.

[2] A. E. Brouwer, A. M. Cohen and A. Neumaier, Distance-Regular Graphs, volume 18 of Ergebnisse der Mathematik und ihrer Grenzgebiete (3), Springer-Verlag, Berlin, 1989, doi: 10.1007/978-3-642-74341-2.

[3] P. J. Cameron and J. H. van Lint, Designs, Graphs, Codes and Their Links, volume 22 of London Mathematical Society Student Texts, Cambridge University Press, Cambridge, 1991, doi:10.1017/cbo9780511623714.

[4] H. C. Chan, C. A. Rodger and J. Seberry, On inequivalent weighing matrices, Ars Comb. 21 (1986), 299-333.

[5] E. Ghasemian and G. H. Fath-Tabar, On signed graphs with two distinct eigenvalues, Filomat 31 (2017), 6393-6400, doi:10.2298/fil1720393g.

[6] M. Harada and A. Munemasa, On the classification of weighing matrices and self-orthogonal codes, J. Comb. Des. 20 (2012), 40-57, doi:10.1002/jcd.20295.

[7] Y. Hou, Z. Tang and D. Wang, On signed graphs with just two distinct adjacency eigenvalues, Discrete Math. 342 (2019), 111615, 8, doi:10.1016/j.disc.2019.111615.

[8] J. McKee and C. Smyth, Integer symmetric matrices having all their eigenvalues in the interval [-2, 2], J. Algebra 317 (2007), 260-290, doi:10.1016/j.jalgebra.2007.05.019.

[9] E. Spence, Regular two-graphs on 36 vertices, Linear Algebra Appl. 226/228 (1995), 459-497, doi:10.1016/0024-3795(95)00158-n.

[10] Z. Stanić, On strongly regular signed graphs, Discrete Appl. Math. 271 (2019), 184-190, doi: 10.1016/j.dam.2019.06.017.

[11] Z. Stanić, Spectra of signed graphs with two eigenvalues, Appl. Math. Comput. 364 (2020), 124627, 9, doi:10.1016/j.amc.2019.124627.

[12] E. R. van Dam and E. Spence, Small regular graphs with four eigenvalues, Discrete Math. 189 (1998), 233-257, doi:10.1016/s0012-365x(98)00085-5. 


\section{A Appendix}

Here we list the inequivalent weighing matrices of weight 5 mentioned in Theorem 2.1. The first parameter denotes the order, and the second one denotes the weight. In the schematic representations we write + for 1 , - for -1 and dot for 0 . For $E(4 t+2,5), F(4 t+4,5)$ we have $t \geq 2$ and every unspecified entry is 0 .
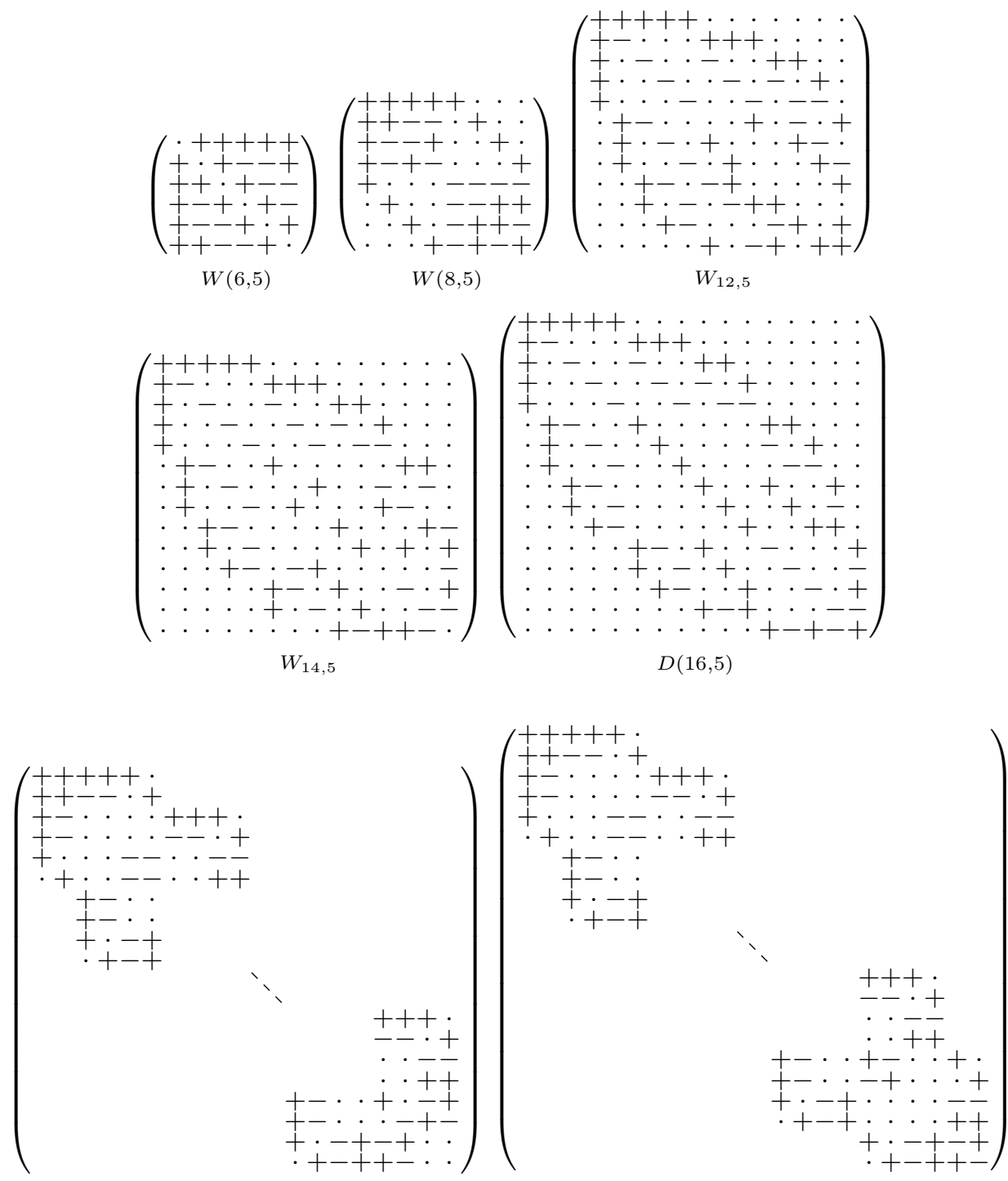

$E(4 t+2,5)$

$F(4 t+4,5)$ 